\title{
Multilevel Task Partition Algorithm for Parallel Simulation of Power System Dynamics
}

\author{
Wei Xue and Shanxiang Qi \\ Department of Computer Science and Technology, Tsinghua University, \\ Beijing, China, 100084 \\ xuewei@tsinghua.edu.cn, qishanxiang@gmail.com
}

\begin{abstract}
Nowadays task partition for parallel computing is becoming more and more important. Particular in power system dynamic simulation, it is critical to design an efficient partition algorithm to reduce the communication and balance the computation load [1]. This paper presents a novel multilevel partition scheme based on the graph partition algorithm. By introducing regional characteristic into the partition, improving the weights of nodes and edges, proposing an objective function to evaluate the partition results and some other schemes, we can efficiently improve the defects in the traditional partition methods. With 12 CPUs for a large scale power system with 10188 nodes, the parallel efficiency with our new algorithm was $63 \%$ higher than that with METIS, a well-known program used for partitioning graphs. The proposed algorithm will satisfy the requirement for large scale power grid dynamic simulation.
\end{abstract}

Keywords: power system, parallel simulation, task partition, graph partition.

\section{Introduction}

Power system dynamic simulation is an important tool in power system research. With the development of power system scale, the computation tasks are becoming heavier and more complex. Up to now, there have been many research topics related to parallel algorithms and parallel simulation of power system dynamics [2-10]. To accomplish the task of power system dynamic simulation, a set of differential algebraic equations (DAEs) have to be solved.

$$
\left\{\begin{array}{l}
\dot{\mathbf{X}}=f(\mathbf{X}, \mathbf{V})=\mathbf{A X}+\mathbf{B} u(\mathbf{X}, \mathbf{V}) \\
0=I-Y(\mathbf{X}) * \mathbf{V}
\end{array}\right.
$$

In (1), the first nonlinear differential equation group describes the dynamic characteristics of the power devices, and the second nonlinear equation group represents the restriction of the power network, where $\boldsymbol{X}$ is the state vector of individual dynamic devices, $\boldsymbol{I}$ is the vector of current injected from the devices into the network, $\boldsymbol{V}$ is the node voltage vector, $\boldsymbol{Y}(\mathrm{X})$ is the complex sparse matrix, which is not constant with time, and is the function of $\boldsymbol{X}$ and $\boldsymbol{V}$.

The most commonly used sequential algorithm for parallel simulation of power system dynamics is the interlaced alternating implicit approach (IAI algorithm). The 
IAI algorithm uses a trapezoidal rule in the difference process and solves differential equations and algebraic equations alternately and iteratively. It not only maintains the advantages of the implicit integration approach, but also has modeling and computing flexibility.

Now the time consumed for simulation increases super-linearly as the power system's size increases. The performance of sequential simulation is not adequate for real-time simulation of large scale power grids, which is critical for power system planning and dispatching. Its limitations become increasingly serious as the integrated power grid continues to develop. Therefore, it is very important to study practical parallel algorithms and software.

The key problem of solving the equations is to solve a sparse linear system, which can be briefly formulated as follows:

$$
\left[\begin{array}{ll}
\boldsymbol{Y} & M^{\prime} \\
M & Z_{C F}
\end{array}\right]\left[\begin{array}{l}
U \\
I
\end{array}\right]=\left[\begin{array}{c}
I_{p} \\
0
\end{array}\right]
$$

In which,

$$
\begin{aligned}
& \mathbf{M}=\left[\begin{array}{llllll}
\mathbf{M}_{C F-1 p} & \mathbf{M}_{C F-2 p} & & & & \\
& & \mathbf{M}_{C F-1 n} & \mathbf{M}_{C F-2 n} & & \\
& & & & \mathbf{M}_{C F-1 z} & \mathbf{M}_{C F-2 z}
\end{array}\right] \\
& \boldsymbol{Y}=\left[\begin{array}{cccccc}
\boldsymbol{Y}_{1 p} & & & & & \\
& \boldsymbol{Y}_{2 p} & & & & \\
& & \boldsymbol{Y}_{1 n} & & & \\
& & & \boldsymbol{Y}_{2 n} & & \\
& & & & \boldsymbol{Y}_{1 z} & \\
& & & & & \boldsymbol{Y}_{2 z}
\end{array}\right] \mathbf{U}=\left[\begin{array}{c}
\mathbf{U}_{1 p} \\
\mathbf{U}_{2 p} \\
\mathbf{U}_{1 n} \\
\mathbf{U}_{2 n} \\
\mathbf{U}_{1 z} \\
\mathbf{U}_{2 z}
\end{array}\right] \\
& \boldsymbol{I}_{p}=\left[\begin{array}{c}
\boldsymbol{I}_{1 p} \\
\boldsymbol{I}_{2 p} \\
0 \\
0 \\
0 \\
0
\end{array}\right] \boldsymbol{M}^{\prime}=\left[\begin{array}{lll}
\boldsymbol{M}_{1 p-C F} & & \\
\boldsymbol{M}_{2 p-C F} & & \\
& \boldsymbol{M}_{1 n-C F} & \\
& \boldsymbol{M}_{2 n-C F} & \\
& & \boldsymbol{M}_{1 z-C F} \\
& & \boldsymbol{M}_{2 z-C F}
\end{array}\right] \\
& \mathbf{Z}=\mathbf{Z}_{C F}, \mathbf{I}=\mathbf{I}_{C F}
\end{aligned}
$$

Where the subscripts 1 and 2 represent the sub-area number, and the subscripts $\mathrm{p}, \mathrm{n}, \mathrm{z}$ represent the positive, negative, and zero sequence networks respectively. $\mathbf{Y}_{\mathbf{1 p}}, \mathbf{Y}_{\mathbf{2 p}}$, $\mathbf{Y}_{1 \mathbf{n}}, \mathbf{Y}_{\mathbf{2 n}}, \mathbf{Y}_{1 \mathrm{z}}$ and $\mathbf{Y}_{\mathbf{2 z}}$ are the admittance matrices of three (positive, negative and zero) sequence networks respectively. $\mathbf{Z}_{\mathbf{C F}}$ is the impedance matrix for cutting branches (the branches between different sub-areas) and fault branches, and also the coefficient matrix of boundary equations in the BBDF computation. $\mathbf{M}$ and $\mathbf{M}$ ' are the associated matrices between $\mathbf{Y}$ and $\mathbf{Z}$ respectively.

As shown in (2), the equations have Block Bordered Diagonal Form and can match the concept of parallelism. Each sub-area calculates its part of the solution. But obviously, each sub-area should calculate in serial with the boundary system represented by $\mathbf{Z}$. Global communication like gather/scatter is used between the 
processes of sub-areas and boundary system. Meanwhile, the boundary system is formed due to the connections between sub-areas. So the more connections between sub-areas, the larger boundary system as well as the more communication between processes in parallel simulation.

It is an essential and critical step to appropriately partition the power system so as to map the computational load onto the processors. Generally, there are two main objectives for the partition:

A. Minimization of the connections between different sub-areas;

B. Maintain an equal computational load for each sub-area.

Power systems are large scale networks with connections related to geographical positions. The problem of network decomposition can be changed into the graph partition problem, which is well developed in HPC field [12]. However, if a graph partition algorithm is simply used; more communication time will be introduced into the parallel simulation because of more cutting edges during graph partitioning [13]. On the other hand, because power systems are developed with connections of regional networks, the connections in the regional network are much tighter than those between regions. So partitioning based on this regional characteristic of power networks can reduce the communication cost in parallel simulation, but it may introduce more load imbalance.

Based on the observation above, this paper presents a novel multilevel partition scheme, which integrates the advantages of graph partition algorithms and the partition method based on power network regional characteristics. This scheme efficiently reduces the boundary system computation as well as the communication in power system dynamic simulation.

\section{Multilevel Partition Algorithm}

There are four main steps in our new scheme as shown by Fig 1:

1) Establish the layered regional model for the power network. This model indicates the weak connections in a power network and gives the guide for successive partition. Thank to the development of power system, we can get the model easily by its obvious regional nature.

2) Network coarsing. During the coarsing phase, aggregating the nodes based on the reasonable level (such as the highest level) of regional model successively decreases the size of the power network graph. The partition problem of power networks is formulated into a weighted graph partition problem, in which the weights of the vertex and edge represent the computation load of the sub-network in the vertex and the amount of communication between sub-networks. Following network coarsing, the multilevel partition algorithm can identify the weak connections in a power network easily and effectively. The time consumed in the partition process is also reduced sharply.

3) Multi-level partition. In the multilevel partitioning phase, the derived small weighted graph is broken down into a specific number of sub-graphs with a graph partition algorithm (bisection method is used here). As we know, the small derived graph may limit the freedom in partitioning and may bring more load 
imbalance into the final solution. The multilevel scheme is, therefore, proposed to evaluate the quality of partition results, decompose the sub-area with the maximum computation load and partition the new graph again. This process continues to run until the optimal solution is found.

4) Refine results. After the weighted graph partition, the results have to be applied to the original network. Further refinement and adjustment are performed to improve the quality of final partition results.

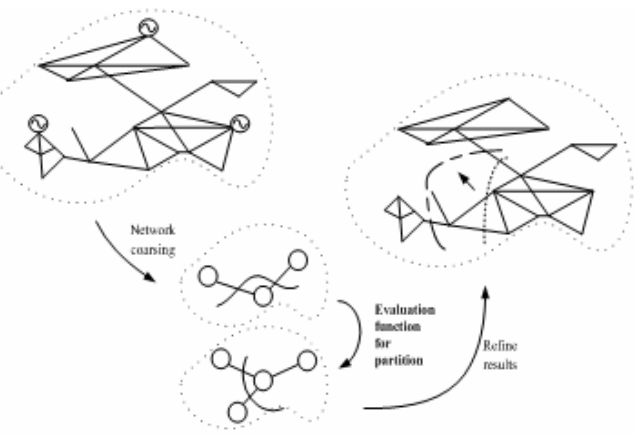

Fig. 1. The process of multilevel partition algorithm

The following key points in the new method can be described as follows:

1) How to construct graph in order to describe the amount of computation and communication.

2) How to evaluate the partition results and then control the partitioning process.

3) How to refine results for partition if possible.

\subsection{Establish the Layered Model}

Due to the nature of the power system, we can establish the layered model according to the regionalism.

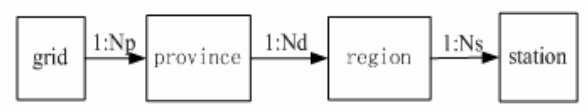

Fig. 2. Layered Power Network Model

\subsection{Weights for Graph Vertex and Edge}

In this paper, we introduce the weights of the vertex and edge to represent the computation load of the sub-network in the vertex and the amount of computation in boundary system. 
Simulation for power system dynamics is floating point computation dominated. So the weight of the vertex describes the amount of floating point computation of the devices and network. So the vertex $V_{i}$ 's weight $W V_{V_{i}}$ is shown below:

$$
W V_{V_{i}}=N_{n}+2 N_{b C}+\sum_{D i \in V D_{i}} \operatorname{Path}\left(D_{i}\right)
$$

In which, $N_{n}$ is the number of the nodes in the coarsing vertex. $N_{b C}$ is the number of the edges between nodes in vertex. $N_{n}+N_{b C}$ represents the floating point computation of network in vertex. $V D i$ is the set of the devices related to $V_{i}$. $\operatorname{Path}\left(D_{i}\right)$ is the estimation of computation for device $D_{i}$, which can be regarded as the number of edges in relation graph between variables for specific device mathematical model.

The weight of the edge describes the contribution of the cutting branches to the boundary system. With our parallel algorithm, the nodes related to the cutting edges will be included in the boundary system. But the size of boundary system is not equal to twice of the number of cutting edges. So the edge $E_{i}$ 's weight $W E_{E_{i}}$ is shown below:

$$
W E_{E_{i}}=\frac{1}{D E\left(N_{p}\right)}+\frac{1}{D E\left(N_{q}\right)}
$$

In which, $D E\left(N_{p}\right)$ and $D E\left(N_{q}\right)$ are the degree of the nodes. This weight can give an accurate estimation of size of boundary system, which is critical for BBDF parallel algorithm.

\subsection{Evaluation Function for Partition}

Our evaluation function is based on the implementation of BBDF parallel algorithm.

$$
F(p)=\underset{i=1, \ldots, p}{\operatorname{ax}}\left(\operatorname{Comp}_{\operatorname{Cost}}\right)+\operatorname{CompCost}_{\text {Border }}
$$

In which CompCost is $_{i}$ the computation of the sub-area, and CompCost Border $_{\text {is }}$ is the computation of the boundary system described below. In the evaluation function, the

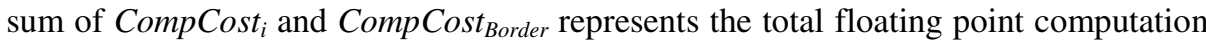
in the critical path and almost the overall time consumed in the parallel simulation. It is noted that the more communication occurs between subtasks, the more computation is introduced into the boundary system. So the influence of communication has been taken into account by CompCost Border $_{\text {. }}$

According to this problem, we suggest that:

$$
\begin{gathered}
\text { CompCost }_{i}=V W_{i}+N_{T i}^{2}+2 * \sum_{j \in T i} \operatorname{Degree}(j) \\
\text { CompCost }_{\text {Border }}=\sum_{i=1}^{p} N_{T i}^{2}+N_{C}
\end{gathered}
$$


In which $\boldsymbol{V} \boldsymbol{W}_{\boldsymbol{i}}$ is the floating point computation of the internal network (computation related to $\boldsymbol{Y}$ in formula 2), which can be evaluated by formula 3. $\boldsymbol{N}_{T i}$ is the number of nodes included in boundary system. An interface matrix has to be computed by subarea $i$ and be communicated to boundary system. Due to the interface matrix is almost dense, the computation can be evaluated by the square of the matrix dimension $N_{T i}$. And the computation related to $\mathrm{M}$ and $\mathrm{M}^{\mathrm{T}}$ in formula 2 in sub-area $i$ is about the degree of nodes, which related to boundary system. With the same idea, we can get the computation evaluation of boundary system using formula 7.

In fact, it is difficult to give the accurate computation overhead in sparse matrix operation before factoring. Based on our analysis and testing, for comparing the different partition results, our method gives a trade-off between accuracy and speed to evaluate the overhead of parallel computation effectively.

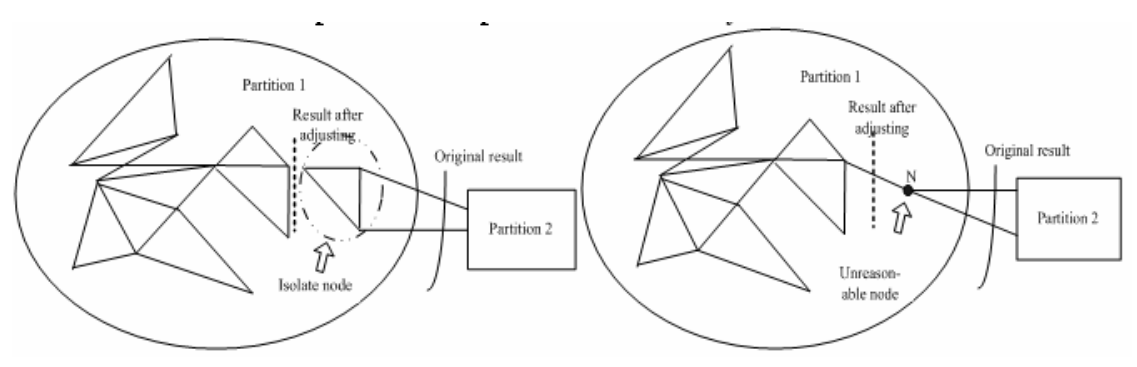

Fig. 3. Partition improvement for isolated node set

Fig. 4. Partition improvement for Unreasonable node

\subsection{Refine Results}

After the graph partitioning phase, it may have two similar problems: isolate node sets and unreasonable node related to the cutting branches. These two problems are shown above.

We identify the isolated node sets by topological analysis and analyze the connections of the nodes in the boundary system to improve the quality of final partition results.

\section{Test Results}

In this study, two power systems shown in Table 1 have been tested on a SMP Cluster. In the cluster, each node is a SMP computer and has 4 Intel Xeon PIII700MHz CPUs and 1 gigabyte of memory. The communication device between SMP nodes is Myrinet with a bandwidth of $2.56 \mathrm{~Gb} / \mathrm{s}$. The software environments are Redhat Linux 7.2 (kernel version 2.4.7-10smp), MPICH-1.2.1..7 and gm-1.5pre4.

The detail computation models including 5 order generator model, typical exciter and governor model, and induction motor model, are concerned [13] for all test cases. An A-phase fault on single $220 \mathrm{kV}$ branch is assumed for Case 1, the fault occurs at $0 \mathrm{~s}$, and the branch trips at $0.16 \mathrm{~s}$. It is a typical non-symmetric fault case. A threephase fault is assumed for Case 2, the fault occurs at 0s, and the branch trips at $0.08 \mathrm{~s}$. 
It is a serious symmetric fault case with numerical difficult. In the simulations, the fixed time step $0.01 \mathrm{~s}$ is used, and the simulation time is $10 \mathrm{~s}$. The convergence tolerance is $10^{-4} \mathrm{pu}$. For all the test cases, the same code for parallel algorithm and complier flags are used as well as the code tuning schemes.

Table 1. Network information for two power systems

\begin{tabular}{cccc}
\hline & System & Case 1 & Case 2 \\
\hline \multirow{3}{*}{ Scale } & number of nodes & 706 & 10188 \\
& number of branches & 1069 & 13499 \\
& number of generators & 88 & 1072 \\
& number of loads & 459 & 3003 \\
\hline
\end{tabular}

In the figures below, $\mathrm{Sp}$ stands for speedup, which is the ratio of the time required for parallel simulations with partitions to the time required for sequential simulations without partitions. And the efficiency is expressed as $\mathrm{E}=\mathrm{Sp} / \mathrm{P}$, where $\mathrm{P}$ is the number of CPUs.

For Case 1, experiment result shows the maximum deviation of node voltage with the parallel program presented in this paper and the software named PSASP. PSASP is the standard sequential software package developed by EPRI China, which is widely used for power system simulation in China [14, 18]. According to Fig.5, the maximum deviation of node voltage between our algorithm and PSASP was less than $10^{-5} \mathrm{pu}$. This proves that our algorithm is accurate.

Table 2 shows that the multilevel partition scheme results in much fewer cutting edges than the recursive bisection algorithm in METIS [15] but leads to a little more imbalance between sub-areas. In the table, the "Max/Min" represents the ratio of the number of nodes in the maximal partition to the number in the minimal partition, and "CutBrn" represents the number of branches between sub-areas.

Table 2. Comparison of the multi-level scheme and algorithm in METIS (Case 1)

\begin{tabular}{ccccc}
\hline \multirow{2}{*}{$\begin{array}{c}\text { Partition } \\
\text { number }\end{array}$} & \multicolumn{2}{c}{ Multilevel scheme } & \multicolumn{2}{c}{ METIS algorithm } \\
\cline { 2 - 5 } & Max/Min & CutBrn & Max/Min & CutBrn \\
\hline 2 & $422 / 284$ & 4 & $379 / 327$ & 12 \\
4 & $301 / 121$ & 7 & $207 / 156$ & 28 \\
8 & $119 / 68$ & 34 & $145 / 74$ & 54 \\
\hline
\end{tabular}

Following experiments suggest that the multilevel scheme achieves higher efficiency in power system dynamic simulations on cluster systems, and its performance does not noticeably suffer from the imbalance of sub-areas. Furthermore, higher efficiency is achieved with the increase of partition number. With eight CPUs, the efficiency of our algorithm was about $70 \%$ higher than that of METIS. 
Table 3. Comparison of the multi-level scheme and algorithm in METIS (Case 2)

\begin{tabular}{ccccc}
\hline \multirow{2}{*}{$\begin{array}{c}\text { Partition } \\
\text { number }\end{array}$} & \multicolumn{2}{c}{ Multilevel scheme } & \multicolumn{2}{c}{ METIS algorithm } \\
\cline { 2 - 5 } & Max/Min & CutBrn & Max/Min & CutBrn \\
\hline 2 & $5258 / 4930$ & 2 & $5102 / 5086$ & 2 \\
4 & $3205 / 762$ & 6 & $2552 / 2535$ & 18 \\
8 & $2004 / 628$ & 9 & $1284 / 1266$ & 64 \\
10 & $1374 / 762$ & 19 & $1035 / 1008$ & 78 \\
12 & $1143 / 694$ & 27 & $861 / 835$ & 79 \\
16 & $1116 / 305$ & 42 & $644 / 621$ & 121 \\
20 & $1088 / 156$ & 40 & $531 / 496$ & 140 \\
\hline
\end{tabular}

Table 3 also shows that the multilevel partition scheme gets much less cutting edges than the recursive bisection algorithm in METIS, but leads to more imbalance between sub-areas.

With 12 CPUs, the efficiency of our algorithm is about $63 \%$ higher than that of METIS. Besides, we can also find that if the partition number is small, our method is not obviously more efficient than that of METIS. The reason is that the METIS considers the graph containing 10188 nodes in Case 2 for partition, while our method uses the evaluation function to decompose the vertex in a simplified graph. Therefore, in the cases with the small partition number, METIS has more freedom than our method. But in contrast, in the cases with large partition number, our method is better than METIS because of considering the accurate behaviour in the parallel simulation.

In conclusion, the multilevel partition scheme fits cluster-based BBDF algorithm well, especially when more CPUs are used. The multilevel partition scheme and the BBDF algorithm give an integrated solution to parallel dynamic simulation and can get very satisfying results.

Acknowledgements. This work was supported in part by the National Natural Science Foundation of China (Grant No.90612018).

\section{Reference}

1. P. Zhang, J. R. Martí, et al, Network Partitioning for Real-time Power System Simulation. International Conference on Power Systems Transients, No.177

2. W. Xue, J. W. Shu, et al, Advance of parallel algorithm for power system transient stability simulation, Journal of system simulation, 2002, 14(2), 177-182.

3. IEEE Committee Report. Parallel processing in power systems computation. IEEE Trans. on Power Systems, 1992, 7(2): 629-638.

4. I.C.Decker, D.M.Falcao, et al. Conjugate gradient methods for power system dynamic simulation on parallel computers. IEEE Trans. on PWRS, 1996,11(3), 1218-1227

5. M. La Scala, G. Sblendorio, A. Bose, J. Q. Wu. Comparison of algorithms for transient stability simulations on shared and distributed memory multiprocessors. IEEE Trans. on Power Systems, 1996, 11(4), 2045-2050. 
6. G. Aloisio, M. A. Bochicchio, et al. A distributed computing approach for realtime transient stability analysis. IEEE Trans. on Power Systems, 1997, 12(2), 981-987.

7. K.W.Chan, R.C.Dai, C.H.Cheung. A coarse grain parallel solution method for solving large set of power systems network equations. 2002 international conference on power system technology, 2002.Volume: 4: 2640 -2644

8. A. H. Jorge, R. M. Jose Real time network simulation with PC-Cluster. IEEE Trans. on power systems, 2003, 18(2), 563-569.

9. Y. L. Li, X. X. Zhou, Z. X. Wu, Parallel algorithms for transient stability simulation on PC cluster. PowerCon 2002, vol.3, 1592-1596.

10. Y. L. Li, et al, A Parallel Complex Fault Computation Algorithm for Large Scale Power System Digital Simulation. Proceedings of CSEE, 2003, 23(12), 1-5

11. H.Simon, S. Teng. How good is recursive bisection? SIAM J. Scientific Computing, 1997, 18(5):1445-1463

12. Kirk Schloegel, George Karypis, Vipim Kumar. Graph Partitioning for High Performance Scientific Simulations. CRPC Parallel Computing Handbook. Morgan Kaufmann, 2000

13. Jiwu Shu, Wei Xue, Weimin Zheng, An Optimal Partition Scheme of Transient Stable Parallel Computing in Power System, Automation of Electric Power Systems, 2003, 27(19), 6-10.

14. EPRI China, Power System Analysis Software Package, Fundamental database user manual, 2001.

15. K. George, V. P. Kumar, METIS-a software package for partitioning unstructured graphs, partitioning meshes, and computing fill-reducing orderings of sparse matrices, version 4.0 [EB/OL], http:// www.cs.umn.edu/?karypis, 1998,9.

16. M.H.M.Vale, D.M.Falcao, E.Kaszkurewicz, Electrical power network decomposition for parallel computations. Proceedings of the IEEE Symposium on Circuits and Systems. San Diego, CA, May 1992. 2761-2764

17. Banerjee.P., Jones.M.H., Sargent J.S.. Parallel simulated annealing algorithms for cell placement on hypercube multiprocessors. IEEE Trans.,1990,PDS-1:91-106

18. Cheng Hua, Xu Zheng, Comparison of mathematical models for transient stability calculation in PSASP and PSS/E and corresponding calculation results, Power system teconology, 2004, 28(5), 1-4. 